\title{
Curso de Infraestrutura de Redes nas Escolas: Relato de uma Ação do Programa de Educação Tutorial
}

\author{
Nayara Maria Costa de Mesquita ${ }^{1}$, Joao Victor Oliveira Farias ${ }^{1}$, \\ Letícia Saraiva Chaves $^{1}$, Carla Ilane Moreira Bezerra ${ }^{1}$ \\ ${ }^{1}$ Universidade Federal do Ceará - Campus Quixadá \\ Quixadá - CE - Brasil \\ \{nayaramesquit, victorfarias.new, leticia.chave, carlailane\}@gmail.com
}

\begin{abstract}
This article describes the practices developed by the Network Infrastructure Course in Schools, held in a high school. The action was carried out by the Tutorial Education Program (PET). This activity has as main objectives to arouse the interest of high school students in the area of Information Technology (IT) and to promote specific training in activities related to infrastructure and configuration of computer networks through classes that use strategies of active methodologies such as Learning Based on Teams and Dialogue Exposed Classes to disseminate practices common to this field.

Resumo. Este artigo descreve as práticas desenvolvidas pela ação do Curso de Infraestrutura de Redes nas Escolas, realizada em uma escola de ensino médio. A ação foi realizada pelo Programa de Educação Tutorial (PET) e teve como objetivos principais, despertar o interesse dos alunos do ensino médio pela área de Tecnologia da Informação (TI) e promover formação específica em atividades relacionadas a infraestrutura e configuração de redes de computadores através de aulas que utilizam de estratégias de metodologias ativas como Aprendizagem Baseada em Times e Aulas Expositivas Dialogadas para disseminar práticas comuns a esse campo de atuação.
\end{abstract}

\section{Introdução}

As redes de computadores e a Internet ${ }^{1}$ estão atuando nos mais diversos segmentos da sociedade, integrando-se em aspectos que vão desde o ambiente empresarial de negócios, onde a troca rápida de informações se tornou prioridade, até a disseminação de conhecimento e pesquisas por instituições de ensino [Comer 2016].

As tecnologias de telecomunicação também estão fortemente atreladas ao âmbito acadêmico e profissional, atuando como ferramentas de pesquisa e negócios, e principalmente, ditando o rumo do mercado de trabalho, onde se tornaram necessárias para ocupar os mais diversos cargos em setores de Tecnologia da Informação (TI), que vem em uma crescente necessidade por profissionais qualificados na área, e muitas vezes acabam não atendendo a demanda devido à falta de mão de obra qualificada [Vinha 2007].

Analisando esse cenário, percebemos a necessidade de apresentar a esses jovens que estão prestes a ingressar no mercado de trabalho e na área acadêmica, a possibilidade de formação básica em tecnologia de redes de computadores, disseminando conhecimento sobre o funcionamento de estruturas básicas em redes no que diz respeito à sua

\footnotetext{
${ }^{1}$ Internet: Compreende-se por Internet, a rede mundial de computadores.
} 
VIII Congresso Brasileiro de Informática na Educação (CBIE 2019)

Anais do XXV Workshop de Informática na Escola (WIE 2019)

configuração inicial, até a criação de infraestruturas funcionais e interconectadas com a Internet.

Este artigo apresenta um relato de experiência de uma atividade de extensão de ensino de infraestrutura de redes de computadores no ensino médio da rede pública. $\mathrm{O}$ objetivo dessa atividade é despertar o interesse dos estudantes do ensino médio pela área de Tecnologia da Informação (TI), por meio de uma formação voltada para atividades de infraestrutura de redes de computadores e utilizando estratégias inovadoras de ensino.

\section{Fundamentação Teórica}

\subsection{Ensino de Informática com Metodologias Ativas}

No artigo desenvolvido por [Guimarães et al. 2018], é apresentado um relato do impacto de metodologias ativas, como a Aprendizagem Baseada em Times e Sala de Aula Invertida, diretamente ligadas à tecnologias da informação, se tornam extremamente produtíveis para o aprendizado, principalmente à sua dinamicidade que se contrasta com metodologias de ensino clássicas, instigando os alunos participantes do curso a se engajarem nas atividades propostas.

\subsection{Infraestrutura de Redes de Computadores}

De acordo com [Gallo et al. 2003], uma rede de computadores é um coleção de computadores e outros dispositivos eletrônicos que utilizam uma forma de comunicação, também conhecida como protocolo, para compartilhar recursos e informações uns com os outros por intermédio de um meio de transmissão.

Como foi abordado por [Hegering and Lapple 1993], desde a década de sua publicação, os sistemas computacionais deixaram de operar isolados, aumentando o seu poder de processamento e se integrando em compartilhamento de recursos coletivos para a realização de tarefas. A análise de [Hegering and Lapple 1993] é focada no uso de redes locais ( $L A N S$ ) como uma importante infraestrutura operacional baseadas na tecnologia Ethernet ${ }^{2}$ e como sua implementação em ambiente empresarial deve receber atenção.

\section{Metodologia}

Este artigo tem como objetivo apresentar um relato sobre a experiência do ensino de infraestrutura de redes de computadores em uma escola do ensino médio. Neste curso foram utilizadas estratégias de metodologias ativas, para possibilitar maior dinamismo nos processos de ensino e aprendizagem.

Segundo [Souza et al. 2018] através das metodologias ativas é possível estruturar aulas mais dinâmicas e didáticas para apresentar o conteúdo aos alunos. Sendo assim, o plano do curso foi dividido em duas etapas. A primeira foi formada por aulas expositivas dialogadas, práticas e aulas mescladas, sendo esta última parte expositiva e parte prática. Já a segunda etapa foi composta por atividades totalmente práticas que tiveram como principal intuito preparar os estudantes para situações comuns tanto no ambiente acadêmico como no mercado de trabalho.

\footnotetext{
${ }^{2}$ Ethernet: Padrão para meio de transmissão em redes locais.
} 
VIII Congresso Brasileiro de Informática na Educação (CBIE 2019)

Anais do XXV Workshop de Informática na Escola (WIE 2019)

\subsection{Objetivos Principais}

A atividade realizada teve como principais objetivos introduzir o conceito de redes de computadores aos alunos da rede escolar pública, uma das vertentes da área de Tecnologia da Informação (TI), e promover formação técnica específica em atividades que envolviam a montagem de infraestruturas de redes de computadores a partir de métodos inovadores de ensino. O curso procurou tanto capacitar os alunos quanto incentivá-los a ingressarem em cursos do ensino superior ligados a computação por meio de aulas com base em metodologias ativas de ensino.

\subsection{Participantes da Atividade}

O público alvo da atividade foram alunos do ensino médio da rede pública, com pouco ou nenhum conhecimento em redes de computadores ou áreas correlacionadas. As aulas foram ministradas por bolsistas do PET e aconteceram semanalmente no laboratório de informática da escola da rede pública escolhida, com uma turma composta por 19 alunos do Ensino Médio regular e da Educação de Jovens e Adultos (EJA).

\section{Execução do Projeto}

\subsection{Primeira Etapa}

Nos itens abaixo, são apresentados resumos das aulas ministradas na primeira etapa do curso de infraestrutura de redes de computadores.

- Apresentação do curso: Nessa aula, os alunos se apresentaram e comentaram a respeito das suas expectativas com o curso. Também foi apresentada o plano de ensino, a equipe idealizadora da atividade, conceitos iniciais da área de infraestrutura de redes de computadores e carreiras em Tecnologia da Informação(TI).

- Conceitos fundamentais de redes de computadores: Nessa aula, foi abordada a classificação de redes de computadores, a comunicação de dados, os meios de transmissão e as redes sem fio.

- Equipamentos de Redes de Computadores: Nessa aula, foi discutido sobre equipamentos, funcionamento e manutenção de redes de computadores.

- Montagem de cabo de rede: Foram apresentados os padrões de crimpagem 568A e 568B, desenvolvidos pela Electronics Industries Alliance (EIA) e pela Telecomunications Industry Association (TIA), alguns equipamentos e componentes necessários para a atividade, como o decapador de cabos, o alicate de crimpagem, o RJ-45, o cabo par trançado e o testador. Após uma demonstração minuciosa, os alunos foram divididos em grupos e receberam as ferramentas e componentes necessários para realizarem a prática.

- Protocolos e gerência de redes: Nessa aula, foi dada ênfase a apresentação dos protocolos Internet Protocol (IP) e Dynamic Host Configuration Protocol (DHCP). Também foi realizada uma prática de comandos de gerenciamento de redes, onde os alunos conheceram mais sobre comandos de redes, como o ping, ifconfig, tracert e outros.

- Endereçamento e introdução a configuração de redes de computadores: Após a apresentação do protocolo IP, foi discutido como é feito o endereçamento de redes através de uma dinâmica introdutória de configuração de redes, onde foi feita uma disputa simples de alteração do nome da rede solicitada. 
VIII Congresso Brasileiro de Informática na Educação (CBIE 2019)

Anais do XXV Workshop de Informática na Escola (WIE 2019)

Durante as atividades práticas foram formadas duplas ou grupos para proporcionar uma maior trocar de conhecimento entre os alunos. Através desse modelo os estudantes puderam sanar pequenas dúvidas entre si e conseguiram desenvolver os exercícios propostos, além de estimular uma interação entre eles construtiva para o aprendizado.

\subsection{Segunda Etapa}

Para oferecer experiências mais semelhante ao mercado de trabalho e ao ambiente acadêmico, a segunda etapa do curso foi composta por dinâmicas que possibilitaram aprendizagem por meio da resolução de problemas e projetos, em grupos, para possibilitar o aprendizado colaborativo por meio competições amistosas.

Nas simulações, em dado momento os integrantes dos grupos representaram clientes e determinaram as exigências que a rede deveria atender, enquanto os integrantes dos outros grupos representaram técnicos e ficaram responsáveis por garantir a criação e configuração de uma rede que atendesse as preferências solicitadas.

Nas últimas práticas cada grupo teve que entregar uma rede que atendesse os requisitos especificados pelos instrutores em um documento. Os critérios de avaliação consistiram no tempo de entrega da rede e na conformidade dela em relação aos requisitos do projeto. Através dessa atividade os estudantes tiveram a oportunidade de testar seus conhecimentos obedecendo prazos, restrições e interpretando termos técnicos para a consolidação de um serviço.

\section{Resultados}

A turma composta por 19 estudantes foi a primeira a participar do curso de infraestrutura de redes de computadores. Os resultados dessa atividade, realizada pelo PET, são relacionados a avaliação das aulas e o interesse dos estudantes pela área. Os estudantes responderam voluntariamente a um questionário de avaliação, cujo os dados coletados podem ser observados nos gráficos da Figura 1.

As primeiras perguntas feitas no questionário são relacionadas ao nível de conhecimento e interesse dos alunos antes de participarem do curso. Mais da metade dos participantes não sabiam o que era uma rede de computadores $(63,2 \%)$ e a maioria da turma nunca havia configurado uma rede $(89,5 \%)$. Todos os alunos da turma demonstraram interesse em aprender sobre infraestrutura de redes de computadores (100\%).

Todos os alunos (100\%), avaliaram como boas as metodologias adotadas para aulas do curso. A maioria dos participantes qualificaram a velocidade que o conteúdo foi aplicado como ideal $(78,9 \%)$ e as outras respostas relativas à velocidade da abordagem do conteúdo ficaram distribuídas igualmente entre rápida $(10,5 \%)$ e muito rápida $(10,5 \%)$.

As últimas perguntas do questionário são direcionadas à experiência dos alunos após entrarem em contato com a área de redes de computadores. Todos os alunos (100\%) se sentiram estimulados a aprender mais sobre a área, além de considerá-la importante para a sociedade. A maioria dos estudantes também responderam que participariam de algum projeto envolvendo infraestrutura de redes $(84,2 \%)$.

\section{Conclusões finais}

A partir dos resultados, analisamos que a maioria dos participantes do curso nunca haviam configurado ou construído redes de computadores e não tinham conhecimento su- 
VIII Congresso Brasileiro de Informática na Educação (CBIE 2019)

Anais do XXV Workshop de Informática na Escola (WIE 2019)

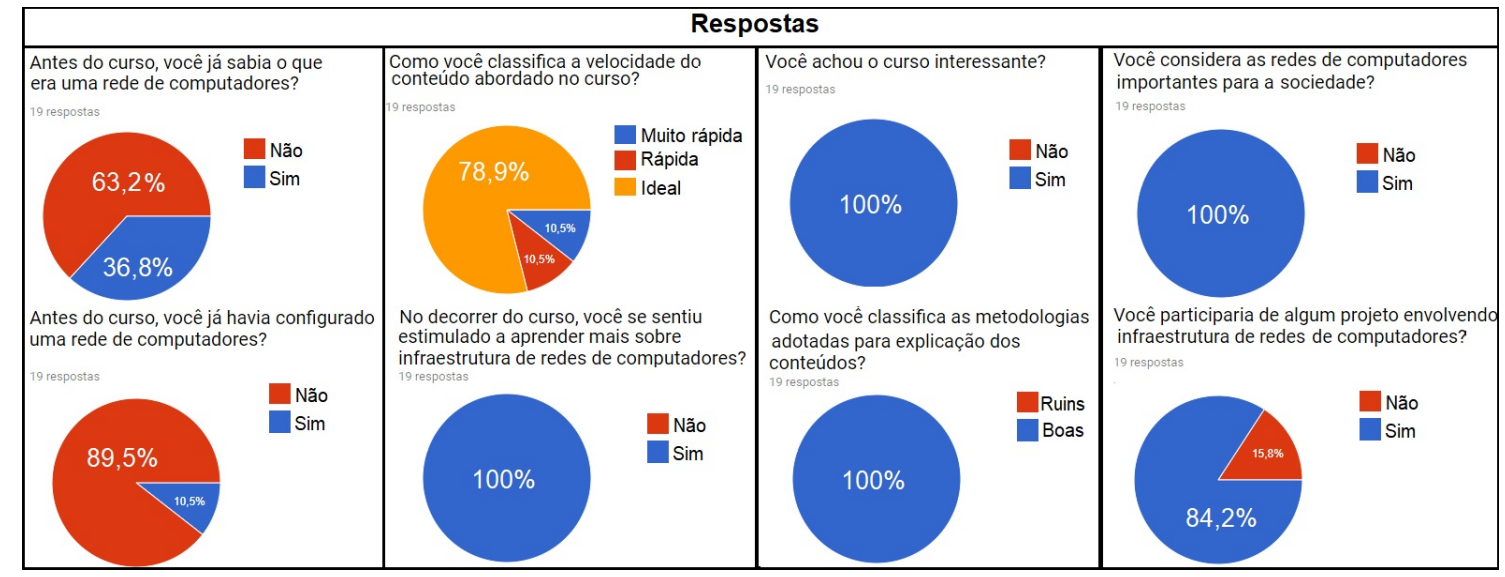

Figura 1. Respostas do questionário

ficiente na área de tecnologia, caracterizando o curso aplicado como uma ferramenta de construção de conhecimentos práticos e habilidades para esses alunos, além de perspectiva profissional, ao mesmo tempo que promove uma atividade engrandecedora aos ministrantes do curso, que junto aos alunos, produziram e compartilharam conhecimento que irá agregar grande valor interpessoal e didático em suas vidas.

Em trabalhos futuros, pretende-se utilizar novas tecnologias e ferramentas de infraestrutura que venham a surgir em Redes de Computadores, como fibras óticas e funções de rede virtuais, sendo uma área que atua em constante inovação e desenvolvimento. Também se pretende utilizar da mesma metodologia com aprimoramentos, visto que a aceitação e aproveitamento desta que foi aplicada, foi unânime.

\section{Referências}

Comer, D. E. (2016). Redes de Computadores e Internet-6. Bookman Editora.

Gallo, M. A., Hancock, W. S., da Silva, F. S. C., de Freitas Carneiro, M. R., and de Melo, A. C. V. (2003). Comunicação entre computadores e tecnologias de rede. Pioneira Thomson Learning.

Guimarães, F., Leite, M., Reinaldo, F., and Ito, G. (2018). Métodos ativos de ensino aliados com tecnologia para a prática de ensino: um relato de experiência. In Anais do Workshop de Informática na Escola, volume 24, page 333.

Hegering, H.-G. and Lapple, A. (1993). Ethernet; Building a Communications Infrastructure. Addison-Wesley Longman Publishing Co., Inc., Boston, MA, USA.

Souza, D., Vergottini, V., and Bernini, D. S. D. (2018). Educação dos tempos modernos através da aprendizagem colaborativa: uma abordagem sobre eduscrum. In Brazilian Symposium on Computers in Education (Simpósio Brasileiro de Informática na Educação-SBIE), volume 29, page 51.

Vinha, T. F. (2007). Tecnologia, trabalho e educação: Perspectivas, estratégias e trajetórias dos jovens no mercado de trabalho informacional. $\mathrm{PhD}$ thesis, Universidade de São Paulo. 